\title{
MicroRNAs in CANCER: MiR-21 and MiR-155
}

\author{
Nnaemeka Darlington Ndodo ${ }^{1,2^{*}}$, Barnabas Danborno ${ }^{3}$, Samuel S Adebisi ${ }^{3}$, \\ Andrew $\mathrm{J} \mathrm{Nok}^{4}$ \\ ${ }^{1}$ Human Anatomy Dept, College of Health Sciences, \\ ${ }^{2}$ Centre for Advanced Medical research \& Training, Usmanu Danfodiyo University, Sokoto, Nigeria. \\ ${ }^{3}$ Human Anatomy Dept, Faculty of Medicine, 4 Biochemistry Dept, Ahmadu Bello University, Zaria, Nigeria \\ *Corresponding Author: Nnaemeka Darlington Ndodo, Human Anatomy Dept, College of Health \\ Sciences, Centre for Advanced Medical research \& Training, Usmanu Danfodiyo University, Sokoto, \\ Nigeria.
}

\begin{abstract}
Cancer has recently surpassed heart disease as the leading cause of death in Americans under the age of 80 years. This staggering statistic is highlighted in the case of lung cancer.

Lung cancer is the leading cause of cancer deaths in the USA with approximately 170,000 newly diagnosed cases each year. The role of miRNAs in various facets of human tumorigenesis,(such as cell proliferation, differentiation, invasion, apoptosis, angiogenesis, migration, and motility) begs the question whether reverting these anomalies of the miRNome can be effectively harnessed for therapeutic gains? Promising preclinical data from many groups encourage this hypothesis and provide the biological enthusiasm for clinical studies in this direction. Re-introduction of miRNAs down-regulated in cancer and/or silencing of miRNAs up-regulated in the tumor may drive apoptosis in cancer cells and lead to a desirable therapeutic outcome.

miR-21 is said to be one of the well characterized mi RNAs and over expressed in various solid tumors including prostate cancers and lung cancers.micro-RNA-21 promoter had been reported to contain highly conserved regions with consensus binding sites for several transcriptional factors, including activator protein 1 (AP-1) and factors of the forkhead family (FOXO), such as FOXO3a.MicroRNA-155 is believed to be encoded in B-cell integration cluster $(B I C)$, a conserved region of the non-coding gene Some generally believe that miR-155 is a master oncogene. This review will focus on these two very important micro RNAs with particular reference to their roles in cancer.
\end{abstract}

Keywords: micro RNA, mi RNA, miR-21, miR-155, cancer

\section{INTRODUCTION}

MicroRNA (miRNA) is a novel family of endogenous small RNA ( 21 nt) present in both plants and animals. The miRNA genes are located often in intergenic regions (between coding genes in the genome) and sometimes in the intron of a coding gene. Like mRNA, miRNA is initially transcribed by RNA polymerase II (pol II) into primary miRNA (pri-miRNA) that is also modified to have a cap structure at the 5' end and a poly (A) tail at the 3 'end.

The pri-miRNAs are processed in the nucleus by the RNase III enzyme, Drosha, and the doublestranded- RNA-binding protein, Pasha (also known as DiGeorge syndrome critical region 8 (DGCR8), into 70-nucleotide pre-miRNAs, which fold into imperfect stem-loop structures. The premiRNAs are then exported into the cytoplasm by the RAN GTP-dependent transporter exportin-5 and undergo an additional processing step in which a double-stranded RNA of $~ 22$ nucleotides in length, referred to as the miRNA: miRNA* duplex, is excised from the pre-miRNA hairpin by another RNAse III enzyme, Dicer (Lagos-Quintana, Rauhut, Meyer, Borkhardt, \& Tuschl, 2003; LagosQuintana, Rauhut, Lendeckel, \& Tuschl, 2001). Subsequently, the miRNA: miRNA*duplex is incorporated into the miRISC complex. Slack and Weidhaas (2006), pointed out the mature miRNA strand is preferentially retained in the functional miRISC complex and negatively regulates its target genes.

\section{MiCRORNAS AND CANCER}

Cancer has recently surpassed heart disease as the leading cause of death in Americans under the age of 80 years. This staggering statistic is highlighted in the case of lung cancer. 
Lung cancer is the leading cause of cancer deaths in the USA with approximately 170,000 newly diagnosed cases each year (Forgacs, Zochbauer-Muller, Olah, \& Minna, 2001). Despite the use of surgery, chemotherapy and radiation in the treatment of lung cancer, the survival rate for patients remains extremely poor. Misregulation of genes that control cell-cycle and cell-fate determination often contributes to cancer.

The role of miRNAs in various facets of human tumorigenesis, (such as cell proliferation, differentiation, invasion, apoptosis, angiogenesis, migration, and motility) begs the question whether reverting these anomalies of the miRNome can be effectively harnessed for therapeutic gains? Promising preclinical data from many groups encourage this hypothesis and provide the biological enthusiasm for clinical studies in this direction. Re-introduction of miRNAs down-regulated in cancer (e.g. miR-15a and miR-16 in BCL2 positive CLL) and/or silencing of miRNAs up-regulated in the tumor (e.g. miR-34 and let-7 in lung cancer) may drive apoptosis in cancer cells and lead to a desirable therapeutic outcome(Slack \& Weidhaas, 2006).

This work would focus on two important microRNAs miR-21 and miR-155 known to be differentially expressed in cancers.

\section{MIR-21 AND CANCER}

According to published sequence data in miRbase, the MIR-21 gene is located in an intergenic region. The length of the gene is reported as 3433 nucleotides long. It overlaps with the 3' UTR end of the Transmembrane Protein 49 (TMEM 49) (also known as Human Vacuole Membrane Protein 1, VMP1). It is suggested that RNA Pol II is the most likely enzyme involved in miRNA transcription. However, studies provide evidences for RNA Pol III dependent transcription of few miRNAs interspersed among repetitive Alu elements.

As reported in the mirBase, the primary transcripts of microRNAs are processed by enzymatic microprocessor Drosha (RNase III enzyme) and DGCR8 (dsRNA binding protein) from their 3' and 5' cleavage sites into an intermediate stem-loop precursor or pre-miRNA in the nucleus.

The precursor of MIR-21 is 72 bases long (pre-MIR-21), forms a secondary structure, and contains the mature miRNA sequence, stem and terminal loop structures with 2-nt 3'overhang The precursor is then transferred from nucleus to cytoplasm by the enzyme Exportin 5. In cytoplasm, a second RNase III enzyme, Dicer, removes terminal loop generating about 20-bp RNA duplex. The length is said to be about 72 bases.

The mature miRNA forms one strand of the RNA duplex. One strand is degraded and other is incorporated in to a protein complex, RNA induced silencing complex (RISC), targeting a partially complementary target mRNA. MIR-21 is 22 nucleotides long.

A number of reports from many groups show that "several tumor suppressors including phosphatase and tensin homolog known to be deleted on chromosome ten (PTEN) (Meng et al., 2007), tumor suppressor gene tropomyosin 1 ( $\mathrm{Zhu}, \mathrm{Si}, \mathrm{Wu}, \& \mathrm{Mo}, 2007$ ), programmed cell death 4 (Asangani et al., 2008; Frankel et al., 2008), maspin (Zhu et al., 2008), and matrix metalloproteinases inhibitors RECK and TIMP3 (Gabriely et al., 2008) were targets of miR-21, the whole data put together suggest that miR-21 is an important oncogenic miRNA which is closely related to tumor growth and metastasis. The expression of miR-21 is also associated according to Blower and others, with prognosis, chemosensitivity and therapeutic outcome in colon Adenocarcinoma" (Blower et al., 2008; Schetter et al., 2008)

Schetter and colleagues particularly stated that they "selected three microRNAs previously implicated in cancer, mir-21, let-7i, and mir-16, and tested the effect of their expression on the potencies of a number of compounds with anticancer activity.

Furthermore, these findings show that those microRNAs may have multiple mechanisms of action. mir-21, in particular, is known to be highly expressed in various cancer types. microRNA functions can be cell context and tissue-dependent. For example, inhibition of mir-21using anti- sense oligonucleotides increased the growth of HeLa cells (derived from cervical cancer) but did not significantly affect the growth of A549 cells (non-small cell lung cancer). Another report showed that inhibition of mir-21 resulted in increased apoptosis in human glioblastoma cells. Other studies have also indicated that mir-21could play a role in drug response. For example, suppression MiR21(Micro-RNA-21 regulates the sensitivity to cisplatin in human neuroblastoma cells as documented by cheng et.al (2012). 
As noted by Cheng et.al,(2012), micro-RNA-21 promoter contains highly conserved regions with consensus binding sites for several transcriptional factors, including activator protein 1 (AP-1) and factors of the forkhead family (FOXO), such as FOXO3a. Activator protein 1, composed of c-Jun and c-Fos family transcription factors, acts upstream of miR-21 and contributes to chemoresistance in cancer stem cell-like side population cells. Again, homodimerization or heterodimerization of epidermal growth factor receptor may activate the ras/ mitogen-activated protein kinase/mitogenactivated protein kinase pathway and then enhance the AP-1 activity and subsequently stimulate the transcription of pri-miR-21. On the other hand, FOXO transcription factor represses the promoter activity of miR-21, and phosphorylated activation of Akt may relieve the repression of the promoter and upregulate the function of miR-21. The targets of miR-21 are also variable. Phosphatase and tensin homolog, a key tumor suppressor that modulates cell growth, division, and death, had been noted to be an important target of miR-21; however, the influence of miR-21 on PTEN is inconsistent in the literature. Micro-RNA-21 had been found to induce downregulation of PTEN and the subsequent up-regulation of cell proliferation and drug resistance in human hepatoma cells and bladder cancer cells (Meng et al., 2007; Tao et al., 2011). As observed by other workers, "the induction of chemoresistance by miR-21 in cancer cells had been demonstrated to be related to the suppression of PTEN and program cell death 4 (PDCD4) expression, enhancement of the phosphoinositide kinase 3/Akt, increased Bcl-2, decreased Bax/Bcl-2 ratio and caspase 3 activity, and reduction of the G2/M arrest and apoptosis (Gong et al., 2011; Bai, Xu, Cao, Wei, \& Wang, 2011; Valeri et al., 2010; Giovannetti et al., 2010; Yang et al., 2013).

MiR-21 is thought to play a significant role in the drug resistance to cisplatin in the neuroblastoma cells. Many reports suggest, miR-21 is important for the resistance to various kinds of drugs in different types of cancer cells, including resistance to doxorubicin in bladder cancer cells (Tao et al., 2011); to trastuzumab, topotecan, or taxol in breast cancer cells (Corcoran, Friel, Duffy, Crown, \& O'Driscoll, 2011; Gong et al., 2011); to arabinosylcytosine or daunorubicin in leukemia cells (Bai et al., 2013; Bai, Xu, Cao, Wei, \& Wang, 2011); to 5-fluorouracil in colorectal cancer cells (Valeri et al., 2010); to interferon $\alpha / 5$-fluorouracil in hepatocellular carcinoma cells (Tomimaru et al., 2010); to VM-26 or temozolomide in glioblastoma cells and to gemcitabine in pancreatic ductal carcinoma cells (Giovannetti et al., 2010).

Liu and workers stated that "miR-21 is one of the well characterized miRNAs and overexpressed in various solid tumors including prostate cancers"(Volinia et al., 2006), (Liu et al., 2011).

MiR-21, in particular, is known to be highly expressed in various cancer types. MicroRNA functions can be cell context and tissue-dependent. For example, Lu and colleagues reported that the inhibition of miR-21using anti- sense oligonucleotides increased the growth of HeLa cells (derived from cervical cancer) but did not significantly affect the growth of A549 cells (non-small cell lung cancer; (Lu et al., 2005). The inhibition of miR-21 resulted in increased apoptosis in human glioblastoma cells.

MiR-21 is thought to play a significant role in the drug resistance to cisplatin in the neuroblastoma cells.

Higher levels of miR-21 are reported in the serum and tumours of patients with cancer. Upon returning miR-21 to endogenous levels the mouse tumours disappeared. Notably, this was the first report indicating the addiction of tumours to a single oncogenic miRNA (termed 'oncomir addiction'). Ubiquitous expression of miR-21, fourfold to sixfold over endogenous levels, resulted in no obvious phenotypes; however, miR-21 overexpression could potentiate the phenotype of mice with a latent KrasG12D allele (KrasLA2), a constitutively activated version of the KRAS proto-oncoprotein. Doubly transgenic animals had an increased lung tumour burden relative to the KrasLA2 mice, but no increase in the rate of conversion from adenoma to adenocarcinoma. By contrast, the lung tumour burden was decreased in KrasLA2; miR-21animals, relative to the KrasLA2 controls. Contrary to earlier report (Hatley et al., 2010), Liu and co-workers (Liu et al., 2011) showed that miR-21 is involved in the later stages of tumorigenesis and not in tumour promotion, as it has no effect on tumorigenesis in the absence of oncogenic KRAS. These studies, combined with human tissue data and cell culture experiments, confirm that miR-21 is an oncogene and provide a rationale for the therapeutic inhibition of miR-21. 
According to Liu and co-workers (Liu et al., 2012), MiR-21 is a miRNA which has been reported to be overexpressed in many human malignancies including Non-small cells lung cancer, NSCLC. In their previous studies, they showed that upregulation of miR-21 in NSCLC serum is correlated with TNM stage and lymph node metastasis (Wang et al., 2011; Fu et al., 2011). Furthermore, the status of serum miR- 21 expressions is found to be an independent prognostic factor for NSCLC patients. They further found that miR- 21 could regulate Adriamycin (ADR) resistance of breast cancer cells, at least in part, by targeting the tumor suppressor gene PTEN. However, the associations of miR-21 expression with malignant phenotypes of NSCLC are still poorly understood.(Stahlhut Espinosa \& Slack, 2006)

\section{MIR-155 AND CANCER}

MicroRNA-155 is encoded in B-cell integration cluster (BIC), a conserved region of the non-coding gene (Medina and Slack, 2008). BIC was originally recognized as a common integration site for the avian leucosis virus, inducing B-cell lymphomas together with MYC (Zhang, Nie, \& Tam, 2008). Overexpression of miR-155 has been observed in both hematological (Eis et al., 2005), (Kluiver et al., 2005) and solid tumors (Zheng et al., 2012). miR-155 is regarded as "masterregulator" of many biological processes. It is a prominent microRNA (miRNA) that regulates genes involved in immunity and cancer-related pathways. MiR-155 is said to be overexpressed in lung cancer, which correlates with poor patient prognosis. It is unclear how miR-155 becomes increased in lung cancers and how this increase contributes to reduced patient survival.

The crucial role of miR-155 is further underscored by independent generation of transgenic, mir-155-overexpressing mice and mir-155-knockout mice which demonstrated that this gene is important in the immune system. Wild-type levels of miR-155 are essential for preserving normal immune system function, including the maintenance of both major classes of cells (B and $\mathrm{T}$ lymphocytes) of the adaptive immune response and dendritic cells, which are involved in the innate immune response. Although mir-155-knockout mice are immunocompromised owing to defects in these cell lineages, overexpression of miR-155 specifically in the B cell lineage results in preleukaemic pre-B cell proliferation in the spleen and bone marrow, followed later in life by B cell malignancy.

\section{REFERENCES}

[1] Asangani, I. A., Rasheed, S. A., Nikolova, D. A., Leupold, J. H., Colburn, N. H., Post, S. et al. (2008). MicroRNA-21 (miR-21) post-transcriptionally downregulates tumor suppressor Pdcd4 and stimulates invasion, intravasation and metastasis in colorectal cancer. Oncogene, 27, 2128-2136.

[2] Bai, H., Wei, J., Deng, C., Yang, X., Wang, C., \& Xu, R. (2013). MicroRNA-21 regulates the sensitivity of diffuse large B-cell lymphoma cells to the CHOP chemotherapy regimen. Int.J.Hematol., 97, 223-231.

[3] Bai,H., Xu, R., Cao, Z., Wei, D., \& Wang, C. (2011). Involvement of miR-21 in resistance to daunorubicin by regulating PTEN expression in the leukaemia K562 cell line. FEBS Lett., 585, 402-408.

[4] Bai,H., Xu, R., Cao, Z., Wei, D., \& Wang, C. (2011). Involvement of miR-21 in resistance to daunorubicin by regulating PTEN expression in the leukaemia K562 cell line. FEBS Lett., 585, 402-408.

[5] Blower,P. E., Chung, J. H., Verducci, J. S., Lin, S., Park, J. K., Dai, Z. et al. (2008). MicroRNAs modulate the chemosensitivity of tumor cells. Mol.Cancer Ther., 7, 1-9.

[6] Chen,Y., Tsai, Y. H., Fang, Y., \& Tseng, S. H. (2012). Micro-RNA-21 regulates the sensitivity to cisplatin in human neuroblastoma cells. J.Pediatr.Surg., 47, 1797-1805.

[7] Corcoran, C., Friel, A. M., Duffy, M. J., Crown, J., \& O'Driscoll, L. (2011). Intracellular and extracellular microRNAs in breast cancer. Clin.Chem., 57, 18-32.

[8] Forgacs, E., Zochbauer-Muller, S., Olah, E., \& Minna, J. D. (2001). Molecular genetic abnormalities in the pathogenesis of human lung cancer. Pathol.Oncol.Res., 7, 6-13.

[9] Frankel, L. B., Christoffersen, N. R., Jacobsen, A., Lindow, M., Krogh, A., \& Lund, A. H. (2008). Programmed cell death 4 (PDCD4) is an important functional target of the microRNA miR-21 in breast cancer cells. J.Biol.Chem., 283, 1026-1033.

[10] Gabriely,G., Wurdinger, T., Kesari, S., Esau, C. C., Burchard, J., Linsley, P. S. et al. (2008). MicroRNA 21 promotes glioma invasion by targeting matrix metalloproteinase regulators. Mol.Cell Biol., 28, 53695380.

[11] Giovannetti, E., Funel, N., Peters, G. J., del, C. M., Erozenci, L. A., Vasile, E. et al. (2010). MicroRNA-21 in pancreatic cancer: correlation with clinical outcome and pharmacologic aspects underlying its role in the modulation of gemcitabine activity. Cancer Res., 70, 4528-4538. 
[12] Gong, C., Yao, Y., Wang, Y., Liu, B., Wu, W., Chen, J. et al. (2011). Up-regulation of miR-21 mediates resistance to trastuzumab therapy for breast cancer. J.Biol.Chem., 286, 19127-19137.

[13] Lagos-Quintana, M., Rauhut, R., Lendeckel, W., \& Tuschl, T. (2001). Identification of novel genes coding for small expressed RNAs. Science, 294, 853-858.

[14] Lagos-Quintana, M., Rauhut, R., Meyer, J., Borkhardt, A., \& Tuschl, T. (2003). New microRNAs from mouse and human. RNA., 9, 175-179.

[15] Liu, L. Z., Li, C., Chen, Q., Jing, Y., Carpenter, R., Jiang, Y. et al. (2011). MiR-21 induced angiogenesis through AKT and ERK activation and HIF-1alpha expression. PLoS.One., 6, e19139.

[16] Lu, J., Getz, G., Miska, E. A., Alvarez-Saavedra, E., Lamb, J., Peck, D. et al. (2005). MicroRNA expression profiles classify human cancers. Nature, 435, 834-838.

[17] Meng, F., Henson, R., Wehbe-Janek, H., Ghoshal, K., Jacob, S. T., \& Patel, T. (2007). MicroRNA-21 regulates expression of the PTEN tumor suppressor gene in human hepatocellular cancer. Gastroenterology, 133, 647-658.

[18] Meng, F., Henson, R., Wehbe-Janek, H., Ghoshal, K., Jacob, S. T., \& Patel, T. (2007). MicroRNA-21 regulates expression of the PTEN tumor suppressor gene in human hepatocellular cancer. Gastroenterology, 133, 647-658.

[19] Schetter, A. J., Leung, S. Y., Sohn, J. J., Zanetti, K. A., Bowman, E. D., Yanaihara, N. et al. (2008). MicroRNA expression profiles associated with prognosis and therapeutic outcome in colon adenocarcinoma. JAMA, 299, 425-436.

[20] Slack, F. J. \& Weidhaas, J. B. (2006). MicroRNAs as a potential magic bullet in cancer. Future.Oncol., 2, 73-82.

[21] Stahlhut Espinosa, C. E. \& Slack, F. J. (2006). The role of microRNAs in cancer. Yale J.Biol.Med., 79, 131-140.

[22] Tao, J., Lu, Q., Wu, D., Li, P., Xu, B., Qing, W. et al. (2011). microRNA-21 modulates cell proliferation and sensitivity to doxorubicin in bladder cancer cells. Oncol.Rep., 25, 1721-1729.

[23] Tao, J., Lu, Q., Wu, D., Li, P., Xu, B., Qing, W. et al. (2011). microRNA-21 modulates cell proliferation and sensitivity to doxorubicin in bladder cancer cells. Oncol.Rep., 25, 1721-1729.

[24] Tomimaru, Y., Eguchi, H., Nagano, H., Wada, H., Tomokuni, A., Kobayashi, S. et al. (2010). MicroRNA21 induces resistance to the anti-tumour effect of interferon-alpha/5-fluorouracil in hepatocellular carcinoma cells. Br.J.Cancer, 103, 1617-1626.

[25] Valeri, N., Gasparini, P., Braconi, C., Paone, A., Lovat, F., Fabbri, M. et al. (2010). MicroRNA-21 induces resistance to 5-fluorouracil by down-regulating human DNA MutS homolog 2 (hMSH2). Proc.Natl. Acad.Sci.U.S.A, 107, 21098-21103.

[26] Volinia, S., Calin, G. A., Liu, C. G., Ambs, S., Cimmino, A., Petrocca, F. et al. (2006). A microRNA expression signature of human solid tumors defines cancer gene targets. Proc.Natl.Acad.Sci.U.S.A, 103, 2257-2261.

[27] Yang, M., Shen, H., Qiu, C., Ni, Y., Wang, L., Dong, W. et al. (2013). High expression of miR-21 and miR-155 predicts recurrence and unfavourable survival in non-small cell lung cancer. Eur.J.Cancer, 49, 604-615.

[28] Zhu, S., Si, M. L., Wu, H., \& Mo, Y. Y. (2007). MicroRNA-21 targets the tumor suppressor gene tropomyosin 1 (TPM1). J.Biol.Chem., 282, 14328-14336.

[29] Zhu, S., Wu, H., Wu, F., Nie, D., Sheng, S., \& Mo, Y. Y. (2008). MicroRNA-21 targets tumor suppressor genes in invasion and metastasis. Cell Res., 18, 350-359.

Citation: N. Darlington Ndodo, "MicroRNAs in CANCER: MiR-21 and MiR-155", International Journal of Research Studies in Biosciences (IJRSB), vol. 5, no. 9, pp. 36-40, 2017. http://dx.doi.org/10.20431/23490365.0509007

Copyright: (C) 2017 Authors. This is an open-access article distributed under the terms of the Creative Commons Attribution License, which permits unrestricted use, distribution, and reproduction in any medium, provided the original author and source are credited. 